\title{
Dosimetric comparison between IMRT and VMAT in irradiation for peripheral and central lung cancer
}

\author{
YI LI $^{1}$, JI WANG $^{2}$, LI TAN $^{3}$, BEINA HUI $^{1}$, XIAOWEI MA ${ }^{3}$, YANLI YAN $^{3}$, CHAOFAN XUE $^{3}$, \\ XIAOTING SHI ${ }^{1}$, EMMANUEL KWATENG DROKOW ${ }^{1}$ and JUAN REN ${ }^{1}$ \\ ${ }^{1}$ Department of Radiotherapy, Oncology Department, The First Affiliated Hospital of Xi'an Jiaotong University, \\ Xi'an, Shaanxi 710061; ${ }^{2}$ Intensive Care Unit, China Meitan General Hospital, Beijing 100028; \\ ${ }^{3}$ Medical School, Xi'an Jiaotong University, Xi'an, Shaanxi 710061, P.R. China
}

Received May 26, 2017; Accepted November 30, 2017

DOI: $10.3892 / \mathrm{ol} .2018 .7732$

\begin{abstract}
The aim of the present study was to compare intensity-modulated radiation therapy (IMRT) and volumetric modulated arc therapy (VMAT) in irradiation of lung cancer. Plans of 14 patients were compared. The results demonstrated that in peripheral lung cancer, V5 (\%) of the lung in partial-arc (PA)-VMAT was decreased compared with IMRT, single-arc (SA)-, and double partial-arc (2PA)-VMAT. V30 (\%) of the lung in IMRT was decreased compared with SA-, PA- and 2PA-VMAT. In the case of planning target volume (PTV) not encompassing the mediastinum in central lung cancer, the conformality index (CI) and heterogeneity index (HI) of SA-VMAT was improved compared with IMRT, PA-, and 2PA-VMAT. The received dose of heart in SA-VMAT was higher compared with IMRT, PA- and 2PA-VMAT. V30 (\%) and V5 (\%) of the lung in IMRT was higher compared with SA-, PA- and 2PA-VMAT; V10 (\%) of the lung in 2PA was decreased compared with IMRT, SA- and PA. In the case of PTV encompassing the mediastinum in central lung cancer, the HI and CI of 2PA was improved compared with IMRT, SA- and PA-VMAT. The received dose of heart in 2PA was higher compared with IMRT, SA- and PA-VMAT. V30 (\%) and V5 (\%) of the lung in 2PA-VMAT was higher compared with IMRT, SA- and PA-VMAT. V20 (\%) of the lung in 2PA was decreased compared with IMRT, SA- and PA-VMAT. In conclusion, it may be necessary to classify the radiotherapy plans of lung cancer into three categories including peripheral lung cancer, PTV not encompassing the mediastinum of central lung cancer, and PTV encompassing
\end{abstract}

Correspondence to: Professor Juan Ren, Department of Radiotherapy, Oncology Department, The First Affiliated Hospital of Xi'an Jiaotong University, 277 Yanta West Road, Xi'an, Shaanxi 710061, P.R. China

E-mail: 869491533@qq.com

Key words: intensity-modulated radiation therapy, volumetric modulated arc therapy, central lung cancer, peripheral lung cancer, dosimetry the mediastinum of central lung cancer. Each of IMRT, SA-VMAT, PA-VMAT, 2PA-VMAT strategy had individual advantages, and therefore it may be crucial to employ different planning techniques for different disease classifications and OAR requirements.

\section{Introduction}

Dose-escalated radiotherapy is an important treatment measure for lung cancers (1-3). Excellent clinical effects have been demonstrated with the treatment of intensity-modulated radiotherapy (IMRT) in conjunction with image-guided radiotherapy (IGRT) (4). IMRT can improve dose conformity, but it requires longer delivery time (5-8). Compared with IMRT, volumetric modulated arc therapy (VMAT) has significantly improved the delivery efficiency and treatment time (7).

IMRT has been previously compared with VMAT in various types of cancer (7,9-19). Several studies have suggested that VMAT produces highly conformal dose distributions, achieves accurate dosimetric delivery and reduces treatment time (7,10,19-23). For lung cancer, IMRT and VMAT are currently used and compared (4,7,10,19-21,23-25). However, little literature exists that focuses on the comparison of IMRT and VMAT in different types of lung cancer, peripheral lung cancer and central lung cancer. In addition, there have been no studies that compare IMRT and VMAT in two different cases in central lung cancer, planning target volume (PTV) encompassing and not-encompassing the mediastinal lymphatic drainage region. In the present study, IMRT and VMAT plans were compared in quality parameters and treatment efficiency in central lung cancer and peripheral lung cancer. Three different plans were employed: Single $360^{\circ}$ arc, part of single $360^{\circ}$ arc and two arcs. Furthermore, IMRT and VMAT plans were compared in central lung cancer in cases of PTV encompassing or PTV not-encompassing the mediastinum.

\section{Patients and methods}

Patients and design. A total of 12 patients with lung cancer, who received radical radiotherapy treatment in the First Hospital Affiliated to Xi'an Jiao Tong University 
(Xi'an, China), 12 patients were enrolled in the study from August 2011 to August 2017. Patients with peripheral lung cancer and central lung cancer were randomly selected. All patients were staged according to the modified 1997 American Joint Committee on Cancer (AJCC) staging system. The patient characteristics were listed in Table I. The present study was approved by the Ethics Committee of the First Hospital Affiliated to Xi'an Jiao Tong University (Xi'an, China) and written consent was obtained from the patients.

PTV delineation and dose prescription. For small cell lung cancer (SCLC), PTV was designed as Gross tumor volume (GTV) plus $1.5 \mathrm{~cm}$ margin, including the ipsilateral hilum and bilateral mediastinum, and excluding the contralateral hilum. For non-NSCLC, GTV encompassed the gross primary tumor and metastatic lymph nodes (LNs) $\geq 1 \mathrm{~cm}$ or hypermetabolic on PET scan. Clinical target volume (CTV) typically encompasses the GTV plus $1-1.5 \mathrm{~cm}$ margin. PTV added a $0.5-0.8 \mathrm{~cm}$ margin on CTV to account for set-up uncertainties and respiratory motion. Respiratory tracking or 4D scanning allow for decreased PTV margins. Each peripheral lung tumor was contoured by two physicians. The PTV of central lung cancer was defined as GTV (including primary cancer and metastatic lymph nodes) plus a margin of $1.5 \mathrm{~cm}$ in lateral, anterior and craniocaudal direction, plus the area within $2 \mathrm{~cm}$ of the proximal bronchial tree, which includes the lower trachea, carina, mainstem bronchi, and the lobar bronchi. Internal target volume (ITV) was used to encompass all mobile tumor positions in breathing cycles for treatment accuracy. For each patient, IMRT plans and VMAT plans were generated simultaneously and compared. 60 Gy was prescribed as the median dose applied to the PTV.

Treatment planning and optimizing IMRT. The IMRT optimization was performed by applying Direct Machine Parameter Optimization (DMPO) algorithm in our treatment planning system (Pinnacle3; Philips Radiation Oncology Systems, Fitchburg, MA, USA), as previously described (13). For each plan, 5 or 7 coplanar beams were used depending on the tumor location. In the plan generation, the maximum iterations and maximum number of all segments in the plan optimizing were 50 and 80. The maximum monitor units (MUs) and segments area were $5 \mathrm{MU}$ and $5 \mathrm{~cm}^{2}$, respectively. Plans were generated for the Elekta Beam Modulator with $10 \mathrm{MV}$.

Single-arc (SA)-VMAT. The VMAT planning was conducted applying the SmartArc planning algorithm in Pinnacle3 9.2 (research version; Philips Radiation Oncology Systems). The optimizer (single arc) was constrained to use one single $360^{\circ}$ arc which consisted of 90 control points. The arc was represented by 89 beams each separated by $4^{\circ}$, which started at $181^{\circ}$ and ended at $180^{\circ}$. The accelerator-used automatic dose rate was selected for each individual segment of the arc. Plans were generated with $10 \mathrm{MV}$.

Partial-arc (PA)-VMAT. The plans were optimized in the same planning system mentioned above. A $180-200^{\circ}$ partial arc was generated for standardization across the studied cased with the range coinciding with the tumor location while
Table I. Characteristics of the patients $(n=12)$.

\begin{tabular}{lc}
\hline Characteristics & Total \\
\hline Age (years) & 54 \\
Median & $40-68$ \\
Range & \\
Sex (no. of patients) & 10 \\
Male & 2 \\
Female & \\
Disease stage & 6 \\
II & 4 \\
IIIa & 2 \\
IIIb & \\
Central or peripheral lung cancer & \\
(no. of patients) & 6 \\
Central & 6 \\
Peripheral & \\
PTV volumes (cm ${ }^{3}$ ) & 95.81 \\
Median & $48.78-203.97$ \\
Range & \\
\hline
\end{tabular}

PTV, planning target volume.

avoiding as much of the contralateral lung as possible, which started between $170-180^{\circ}$ and ended between $0-10^{\circ}$. The arc was represented by $44-49$ beams each separated by $4^{\circ}$.

Dual 2PA-VMAT. The plans were generated with two partial arcs, and each partial arc parameter was the same as the one in the PA-VMAT.

Plan evaluation. The quality of plans was evaluated by three radiation oncologists. Dose volume histograms (DVHs) and the corresponding dose distributions of plans were independently reviewed by each oncologist. A total of $95 \%$ of target volume should be encompassed by $95 \%$ of the prescribed dose. PTV coverage was evaluated by using Dmax, Dmin, Dmean, the heterogeneity index (HI) and the conformality index (CI).

$$
H I=\frac{D_{2 \circ}-D_{98_{\circ}}}{D_{50 \circ}}(26)
$$

D2, D98 and D50\% represent the dose of 2, 98 and 50\% target volume. Values of HI closer to 0 indicate greater dose homogeneity within the volume of PTV, while large values indicate more heterogeneous dose distribution.

$$
C I=\frac{V_{T, \text { ref }}}{V_{T}} \times \frac{V_{T, \text { ref }}}{V_{\text {ref }}}(27)
$$

$V_{T, \text { ref }}=$ volume of target receiving a dose equal to or greater than the reference dose, $V_{T}=$ volume of target, $V_{\text {ref }}=$ volume receiving a dose equal to or greater than the reference dose (treated volume). The closer the value of CI to 1.0, the better the dose conformity.

Statistical analysis. The SAS 21.0 software (SAS Institute Inc., Cary, NC, USA) was used to analyze and compare the 

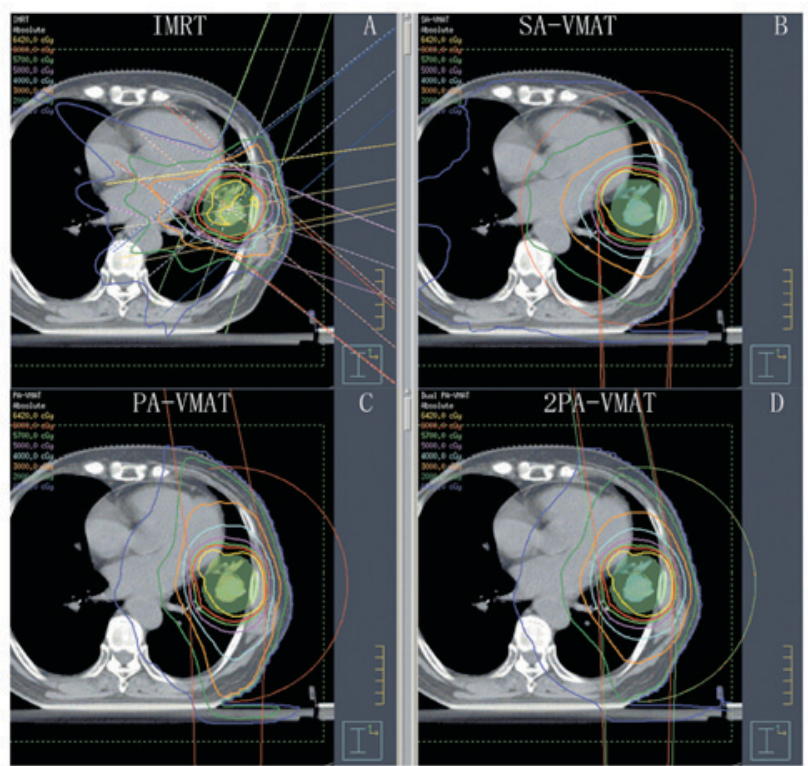

Figure 1. Isodose curves of IMRT plan and 2PA-VMAT plan in peripheral lung cancer. (A) Isodose curves of IMRT plan. (B) Isodose curves of SA-VMAT plan. (C) Isodose curves of PA-VMAT plan. (D) Isodose curves of 2PA-VMAT plan. In peripheral lung cancer, both IMRT plan and VMAT plan exhibited satisfying prescribed tumor target coverage and OAR sparing. However, 2PA-VMAT displayed improved PTV coverage compared with IMRT. 2PA-VMAT exhibited better sparing of spinal cord, lung-all, and lung-contralateral compared with IMRT, while IMRT exhibited better heart and lung-ipsilateral sparing compared with 2PA-VMAT. IMRT had better dose conformity and homogeneity. IMRT, intensity-modulated radiation therapy; VMAT, volumetric modulated arc therapy; 2PA, double partial arc; PA, partial arc; SA, single arc; OAR, organs-at-risk.

data with randomized block design. The data of each treatment group were analyzed with normality test (Shapiro-Wilk test) and homogeneity of variance test (Levene test). Analysis of variance and Fisher's least significant test (LSD-t) were used when data obeyed normal distribution and homogeneity of variance. If the data did not obey the normal distribution, the variance was not uniform, or the data did not obey the normal distribution following conversion, the rank sum test (Friedman's test, also called the M-test) was used for the randomized block design data; if the rank sum test was statistically significant, this indicated that the mean of multiple populations was different or not equal. Then, Friedman's test and multiple comparison non-parametric tests were used. $\mathrm{P}<0.05$ was considered to indicate a statistically significant difference.

\section{Results}

Dosage comparison between VMAT and IMRT plans in peripheral lung cancer. Fig. 1 illustrates the dose-volume histogram (DVH) and dose distributions of IMRT and VMAT plans of a patient with peripheral lung cancer. Figs. 2 and 3 illustrate the CI, HI and organ at risk (OAR) dosage of each patient. The average delivery times for IMRT plans and VMAT plans were 10.5 and 6.1 min respectively. VMAT plans generated larger numbers of MU compared to IMRT.

Table II illustrated the dosage difference between VMAT and IMRT plans in peripheral lung cancer. In peripheral lung cancer, there was no significant difference of CI and HI of PTV

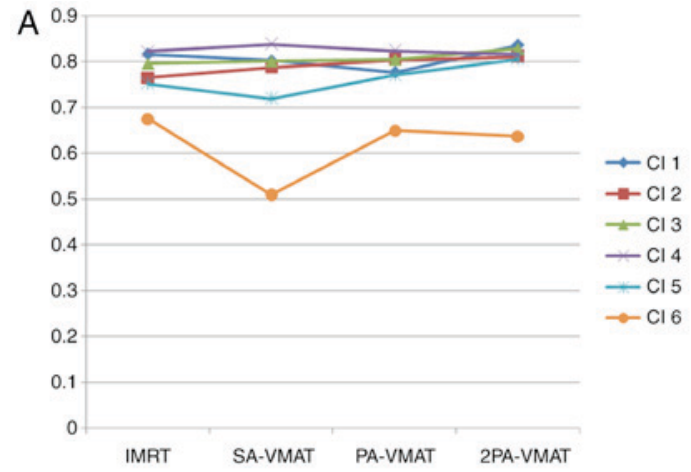

$\mathrm{B}$

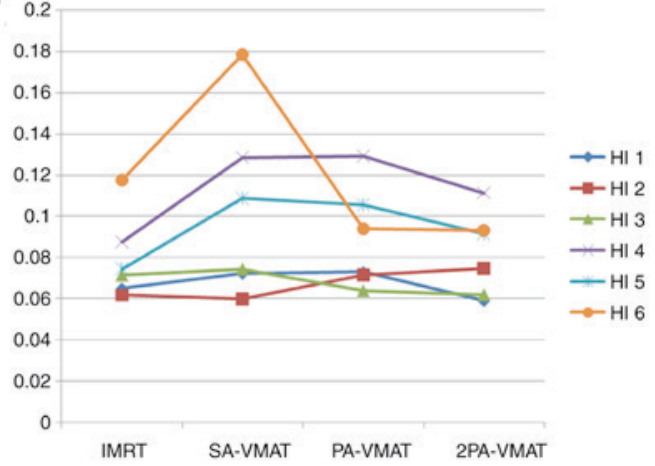

Figure 2. Line charts display the comparison of (A) CI and (B) HI between VMAT and IMRT plans in 6 patients (indicated by different lines) with peripheral lung cancer. The horizontal axis represents the four different plans. The vertical axis represents values of CI and HI. CI, conformity index; HI, homogeneity index; VMAT, volumetric modulated arc therapy; IMRT, intensity-modulated radiation therapy; PA, partial arc; SA, single arc; 2PA, double partial arc.

among IMRT, SA-VMAT, PA-VMAT, and 2PA-VMAT plans (Figs. 1 and 2). The target area is far away from the spinal cord and heart, therefore the received dose of the spinal cord and heart were low; the IMRT plan achieved superior sparing of spinal cord compared with the VMAT plans, and the radiation dose of the spinal cord was the highest in the SA-VMAT plan. IMRT plans exhibited significantly improved sparing of V20, V30 of total lungs and Dmean, V30 of ipsilateral lung compared with SA-VMAT, PA-VMAT and 2PA-VMAT plans. PA-VMAT and 2PA-VMAT exhibited significantly better Dmean, V5 (\%), V10 (\%) of lung-contralateral compared with the IMRT plan. V5 (\%) of lung-all of SA-VMAT was higher compared with PA-VMAT. V5 (\%) of lung-all of IMRT plan was higher compared with SA-, PA- and 2PA-VMAT; V20, V30 (\%) of lung-all of SA-, PA-, 2PA-VMAT were higher compared with IMRT. V5 (\%) of lung-ipsilateral of IMRT was higher compared with SA-, PA-, 2PA-VMAT. IMRT plans also exhibited significantly improved sparing of Dmean of contralateral lung than PA- and 2PA-VMAT plans (Fig. 3).

Dosage comparison between VMAT and IMRT plans in central lung cancer in which the target volume does not encompass the mediastinal lymphatic drainage region. For central lung cancer, it remains unanswered when PTV needs to encompass the mediastinal lymphatic drainage region. It is hypothesized that this depends on the tumor pathological type and whether there exist metastatic mediastinal lymph nodes 
Table II. Dosage comparison between VMAT and IMRT plans in peripheral lung cancer.

\begin{tabular}{|c|c|c|c|c|}
\hline Peripheral lung cancer & $\begin{array}{c}\text { IMRT } \\
(\text { mean } \pm \text { SD) }\end{array}$ & $\begin{array}{c}\text { SA-VMAT } \\
(\text { mean } \pm \text { SD) }\end{array}$ & $\begin{array}{c}\text { PA-VMAT } \\
(\text { mean } \pm \mathrm{SD})\end{array}$ & $\begin{array}{l}\text { 2PA-VMAT } \\
(\text { mean } \pm \text { SD) }\end{array}$ \\
\hline PTV Dmax & $6,654.70 \pm 9.55^{\mathrm{a}}$ & $7,013.00 \pm 7.02$ & $7,042.40 \pm 7.01$ & $6,951.40 \pm 6.83$ \\
\hline PTV Dmin & $5,279.20 \pm 5.22$ & $5,281.40 \pm 5.21$ & $5,151.50 \pm 5.08$ & $5,217.60 \pm 5.17$ \\
\hline PTV D100 (cGy) & $5,279.20 \pm 5.07$ & $5,281.40 \pm 5.01$ & $5,151.50 \pm 5.03$ & $5,217.60 \pm 5.16$ \\
\hline PTV D mean (cGy) & $6,350.00 \pm 6.35^{\mathrm{a}}$ & $6,616.40 \pm 6.11$ & $6,558.70 \pm 6.01$ & $6,560.80 \pm 6.21$ \\
\hline PTV D98 (cGy) & $5,860.00 \pm 5.11$ & $5,790.00 \pm 5.13$ & $57,770.00 \pm 5.07$ & $5,775.00 \pm 5.14$ \\
\hline PTV D95 (cGy) & $6,000.00 \pm 5.56$ & $6,000.00 \pm 5.61$ & $6,000.00 \pm 5.52$ & $6,000.00 \pm 5.59$ \\
\hline PTV D50 (cGy) & $6,387.00 \pm 6.11^{\mathrm{a}}$ & $6,687.00 \pm 6.23$ & $6,654.00 \pm 6.33$ & $6,627.00 \pm 6.22$ \\
\hline PTV D2 (cGy) & $6,557.00 \pm 6.15^{\mathrm{a}}$ & $6,971.00 \pm 6.32$ & $6,967.00 \pm 6.42$ & $6924 \pm 6.25$ \\
\hline Heart V30 $(\%)$ & $8.65 \pm 0.02^{\mathrm{a}}$ & $11.85 \pm 0.03$ & $11.94 \pm 0.03$ & $9.51 \pm 0.02^{\mathrm{c}}$ \\
\hline Heart V40 $(\%)$ & $3.19 \pm 0.00^{\mathrm{a}}$ & $5.73 \pm 0.01$ & $5.62 \pm 0.01$ & $4.55 \pm 0.00$ \\
\hline Spinalcord D max (cGy) & $1,330.3 \pm 1.32^{\mathrm{a}}$ & $1,692.9 \pm 1.43$ & $985.7 \pm 1.01$ & $1,129.7 \pm 1.13$ \\
\hline Spinalcord $\mathrm{D}_{5}$ (cGy) & $1,161 \pm 1.11^{\mathrm{a}}$ & $1586 \pm 1.15$ & $900 \pm 0.91^{\mathrm{b}}$ & $1,025 \pm 1.09^{c}$ \\
\hline Lung all $\mathrm{V} 5^{\mathrm{e}}(\%)$ & $34.93 \pm 0.03^{\mathrm{a}}$ & $37.87 \pm 0.03$ & $22.64 \pm 0.02^{\mathrm{b}}$ & $26.22 \pm 0.02^{c}$ \\
\hline Lung all V10 (\%) & $15.03 \pm 0.01$ & $22.23 \pm 0.01$ & $14.1 \pm 0.00^{\mathrm{b}}$ & $14.59 \pm 0.01^{\mathrm{c}}$ \\
\hline Lung all V20 (\%) & $7.72 \pm 0.00^{\mathrm{a}}$ & $9.63 \pm 0.01$ & $9.17 \pm 0.01$ & $9.21 \pm 0.01$ \\
\hline Lung all V30 (\%) & $5.32 \pm 0.01^{\mathrm{a}}$ & $6.37 \pm 0.01$ & $6.69 \pm 0.01$ & $6.38 \pm 0.01$ \\
\hline Lung all D mean (cGy) & $647.20 \pm 0.61$ & $761.80 \pm 0.71$ & $641.60 \pm 0.62^{\mathrm{b}}$ & $657.30 \pm 0.63^{c}$ \\
\hline Lung-ipsilateral D mean (cGy) & $1,072.40 \pm 1.02^{\mathrm{a}}$ & $1,235.60 \pm 1.21$ & $1,215.20 \pm 1.20$ & $1,209.70 \pm 1.19$ \\
\hline Lung-ipsilateral V5 (\%) & $37.92 \pm 0.03^{\mathrm{a}}$ & $41.74 \pm 0.04$ & $41.49 \pm 0.04$ & $41.64 \pm 0.04$ \\
\hline Lung-ipsilateral V10 (\%) & $30.49 \pm 0.03$ & $33.62 \pm 0.03$ & $32.51 \pm 0.03$ & $33.38 \pm 0.03$ \\
\hline Lung-ipsilateral V20 (\%) & $17.87 \pm 0.01^{\mathrm{a}}$ & $22.22 \pm 0.02$ & $21.22 \pm 0.02$ & $21.31 \pm 0.02$ \\
\hline Lung-ipsilateral V30 (\%) & $12.31 \pm 0.01^{\mathrm{a}}$ & $14.74 \pm 0.01$ & $15.47 \pm 0.01$ & $14.77 \pm 0.01$ \\
\hline Lung-contralateral D mean (cGy) & $323.60 \pm 0.32^{\mathrm{a}}$ & $401.10 \pm 0.04$ & $205.00 \pm 0.02^{\mathrm{b}}$ & $236.90 \pm 0.02^{\mathrm{c}}$ \\
\hline Lung-contralateral V5 (\%) & $32.66 \pm 0.03^{\mathrm{a}}$ & $34.93 \pm 0.03$ & $8.28 \pm 0.00^{\mathrm{b}}$ & $14.48 \pm 0.01^{\mathrm{c}}$ \\
\hline Lung-contralateral V10 (\%) & $3.25 \pm 0.00^{\mathrm{a}}$ & $13.56 \pm 0.00$ & $0.09 \pm 0.00^{\mathrm{b}}$ & $0.28 \pm 0.00^{c}$ \\
\hline Lung-contralateral V20 (\%) & $0.00 \pm 0.00$ & $0.05 \pm 0.00$ & $0.00 \pm 0.00$ & $0.00 \pm 0.00$ \\
\hline Lung-contralateral V30 (\%) & $0.00 \pm 0.00$ & $0.00 \pm 0.00$ & $0.00 \pm 0.00$ & $0.00 \pm 0.00$ \\
\hline HI & $0.11 \pm 0.00^{\mathrm{a}}$ & $0.18 \pm 0.00$ & $0.18 \pm 0.00$ & $0.17 \pm 0.00$ \\
\hline $\mathrm{CI}$ & $0.69 \pm 0.00^{\mathrm{a}}$ & $0.50 \pm 0.00$ & $0.51 \pm 0.00$ & $0.52 \pm 0.00$ \\
\hline
\end{tabular}

${ }^{a} \mathrm{P}<0.05$ compared with $2 \mathrm{PA}-\mathrm{VMAT}$ plan; ${ }^{\mathrm{b}} \mathrm{P}<0.05$ compared with $\mathrm{SA}-\mathrm{VMAT}$ plan; ${ }^{\mathrm{c}} \mathrm{P}<0.05$ compared with $\mathrm{SA}-\mathrm{VMAT}$ plan. IMRT, intensity-modulated radiation therapy; VMAT, volumetric modulated arc therapy; SA, single arc; PA, partial arc; 2PA, double partial arc; $\mathrm{SD}$, standard deviation; PTV, planning target volume; cGy, centigray; HI, homogeneity index; CI, conformity index.

or not. For SCLC, PTV needs to encompass the mediastinal lymphatic drainage region. For NSCLC, if there are metastatic mediastinal lymph nodes, PTV needs to encompass the metastatic lymph nodes. If not, PTV does not encompass it. Fig. 4 illustrates the dose-volume histogram DVH and dose distributions of IMRT and VMAT plans of a patient with central lung cancer whose PTV did not encompass the mediastinum. Figs. 5 and 6 illustrate the CI, HI and OAR dosage of each patient.

In the case of PTV not encompassing the mediastinum in central lung cancer, Table III illustrated the dosage difference between VMAT and IMRT plans. SA-VMAT exhibited significantly superior CI compared with IMRT, PA- 2PA-VMAT. SA- and 2PA-VMAT exhibited significantly superior HI than IMRT and PA-VMAT. Dmax of PTV in SA-VMAT and 2PA was significantly higher compared with IMRT and PA-VMAT. Dmin of PTV in PA-VMAT was significantly lower compared with IMRT and SA-VMAT. V30, V40 (\%) of heart of SA-VMAT were higher compared with IMRT, PA- and 2PA-VMAT in most cases. V5, V10, V20 (\%) of lung-all of IMRT were higher compared with SA-, PA-, and 2PA-VMAT in most cases. V10 (\%) of lung-all of 2PA-VMAT was less compared with IMRT, SA- and PA-VMAT.

Dosage comparison between VMAT and IMRT plans in central lung cancer in which the target volume encompasses the mediastinal lymphatic drainage region. In central lung cancer, in case of either SCLC or positive metastatic mediastinal lymph nodes, PTV needs to encompass the mediastinum. Fig. 7 illustrated the DVH and dose distributions of IMRT and VMAT plans of a patient with central lung cancer whose PTV encompasses the mediastinum. Figs. 8 and 9 illustrate the CI, $\mathrm{HI}$ and OAR dosage of each patient. 

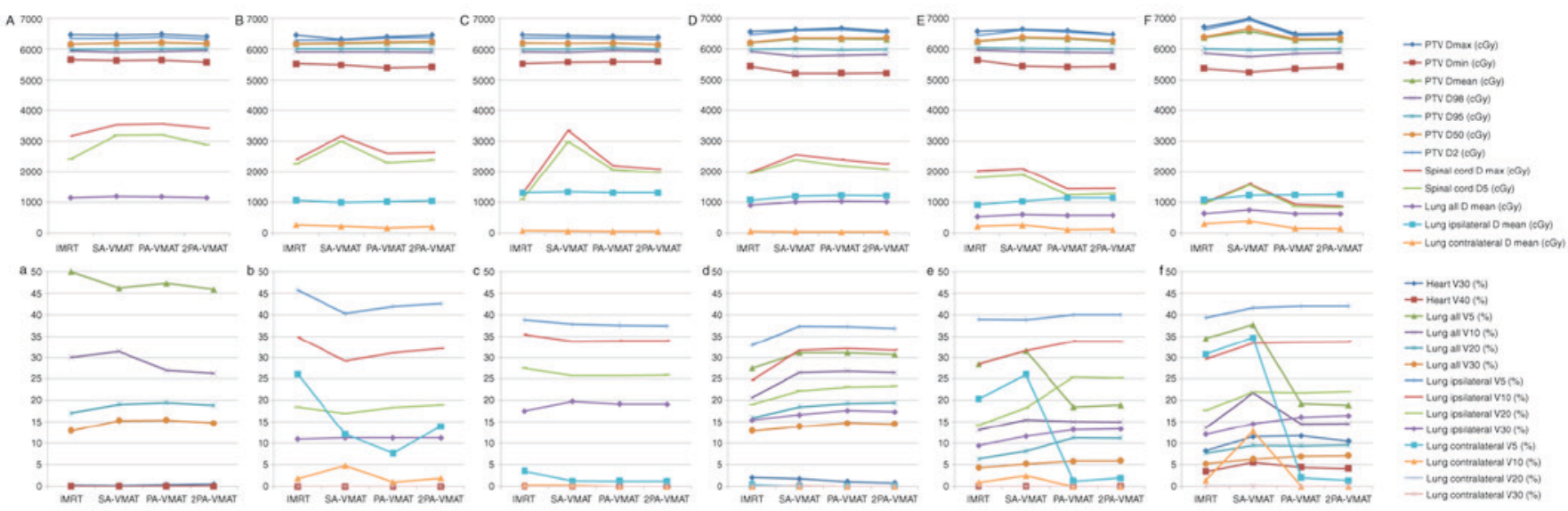

Figure 3. Line charts display the comparison of different dosimetric parameters (indicated by different lines) between VMAT and IMRT plans in 6 patients (indicated by A-a-F-f) with peripheral lung cancer. The horizontal axis represents the four different plans. The vertical axis represents the dose size. For each case, the capital letters show the unit for dose size in cGy, and the small letters show the unit for dose size in \%. VMAT, volumetric modulated arc therapy; IMRT, intensity-modulated radiation therapy; cGy, centigray; PA, partial arc; SA, single arc; 2PA, double partial arc.

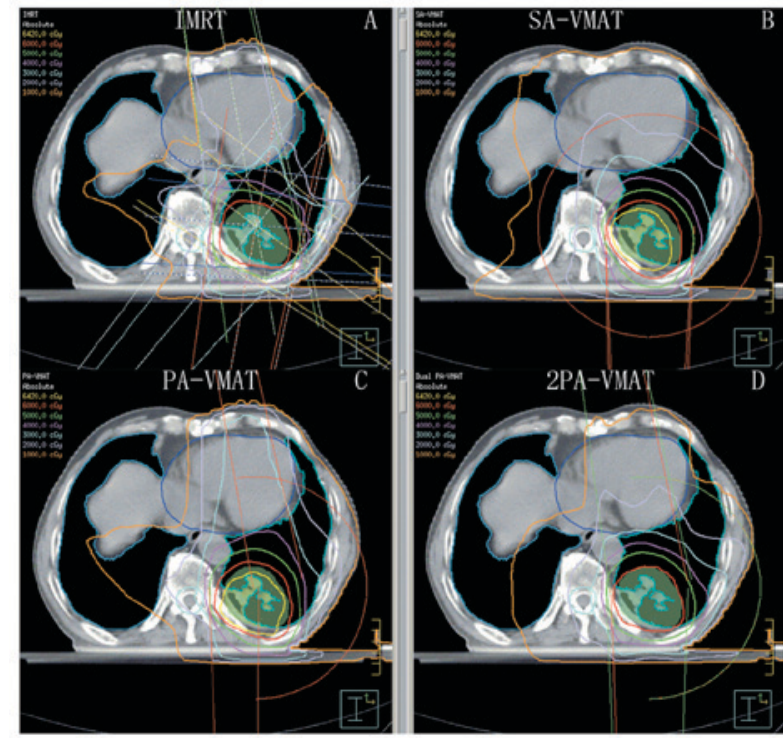

Figure 4. Isodose curves of 2PA-VMAT and IMRT plans in central lung cancer in which the target volume does not encompass the mediastinal lymphatic drainage region. (A) Isodose curves of IMRT plan. (B) Isodose curves of SA-VMAT plan. (C) Isodose curves of PA-VMAT plan. (D) Isodose curves of 2PA-VMAT plan. In central lung cancer, when PTV does not encompass the mediastinum, there was no significant difference in PTV coverage between the IMRT and 2PA-VMAT plans. 2PA-VMAT exhibited better spinal cord sparing, and better dose conformity and homogeneity. 2PA, double partial arc; VMAT, volumetric modulated arc therapy; IMRT, intensity-modulated radiation therapy; PA, partial arc; SA, single arc; PTV, planning target volume.

In the case of PTV encompassing the mediastinum in central lung cancer, Table IV illustrated the dosage comparison between VMAT and IMRT plans. 2PA-VMAT exhibited superior HI and CI compared with IMRT, SA- and PA-VMAT in most cases, but V40 (\%) of heart in 2PA-VMAT was higher compared with IMRT, SA- and PA-VMAT. V5, V30 (\%) of lung-all in 2PA-VMAT was higher compared with IMRT. By contrast, V20 (\%) of lung-all in 2PA-VMAT was less compared with IMRT, SA-, and PA-VMAT. V5, V10 (\%) of lung-contralateral in SA-, PA-, and 2PA-VMAT was higher compared with IMRT, while V20, V30 (\%) of lung-contralateral in 2PA-VMAT was less compared with IMRT.

In conclusion, in peripheral lung cancer, V5 (\%) of the lung in PA-VMAT was less compared with IMRT, SA-, and 2PA-VMAT. V30 (\%) of the lung in IMRT was less compared with SA-, PA- and 2PA-VMAT. In the case of PTV not encompassing the mediastinum in central lung cancer, the CI and HI of SA-VMAT was improved compared with IMRT, PA-, and 2PA-VMAT; the received dose of heart in SA-VMAT was higher compared with IMRT, PA- and 2PA-VMAT. V30 (\%) and V5 (\%) of the lung in IMRT was higher compared with SA-, PA- and 2PA-VMAT; V10 (\%) of the lung in 2PA was less compared with IMRT, SA- and PA-VMAT. In the case of PTV encompassing the mediastinum in central lung cancer, the HI and CI of 2PA was improved compared with IMRT, SA- and PA-VMAT. The received dose of heart in 2PA was higher compared with IMRT, SA- and PA-VMAT. V30 (\%) and V5 (\%) of the lung in 2PA-VMAT was higher compared with IMRT, SA- and PA-VMAT. V20 (\%) of the lung in 2PA was lower compared with IMRT, SA- and PA-VMAT.

\section{Discussion}

The differences of dosimetry and clinical characters between VMAT and IMRT have been studied by many studies (9-16,19,25,27-33) and VMAT technique has displayed superiority in different types of solid tumors compared with IMRT, especially tumors with complicated target volume (1-17). The present study compared dosimetric differences and treatment efficiency between IMRT and three kinds of VMAT plans in the following three situations: Peripheral lung cancer, PTV not encompassing the mediastinal lymphatic drainage region in central lung cancer, and PTV encompassing the mediastinal lymphatic drainage region in central lung cancer. To our knowledge, this is the first study to report dosimetric differences between IMRT, SA-VMAT, PA-VAMT and 2PA-VMAT plans in these three situations.

In peripheral lung cancer, there was no significant difference of CI and HI among IMRT, SA-VMAT, PA-VMAT, and 

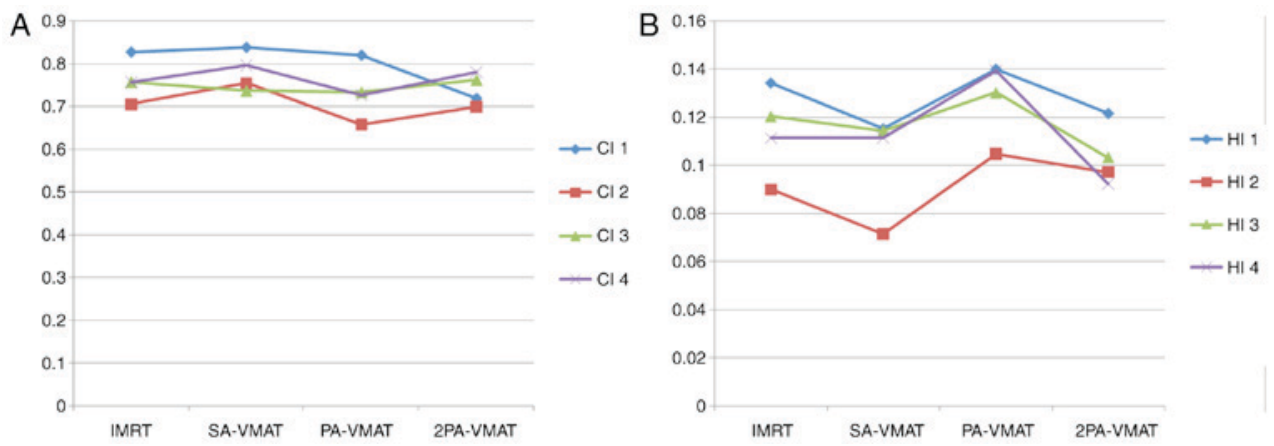

Figure 5. Line charts display the comparison of (A) CI and (B) HI between VMAT and IMRT plans in 4 patients with central lung cancer in which the target volume encompasses the mediastinal lymphatic drainage region. The horizontal axis represents the four different plans. The vertical axis represents values of $\mathrm{CI}$ and HI. CI, conformity index; HI, homogeneity index; VMAT, volumetric modulated arc therapy; IMRT, intensity-modulated radiation therapy; PA, partial arc; SA, single arc; 2PA, double partial arc.
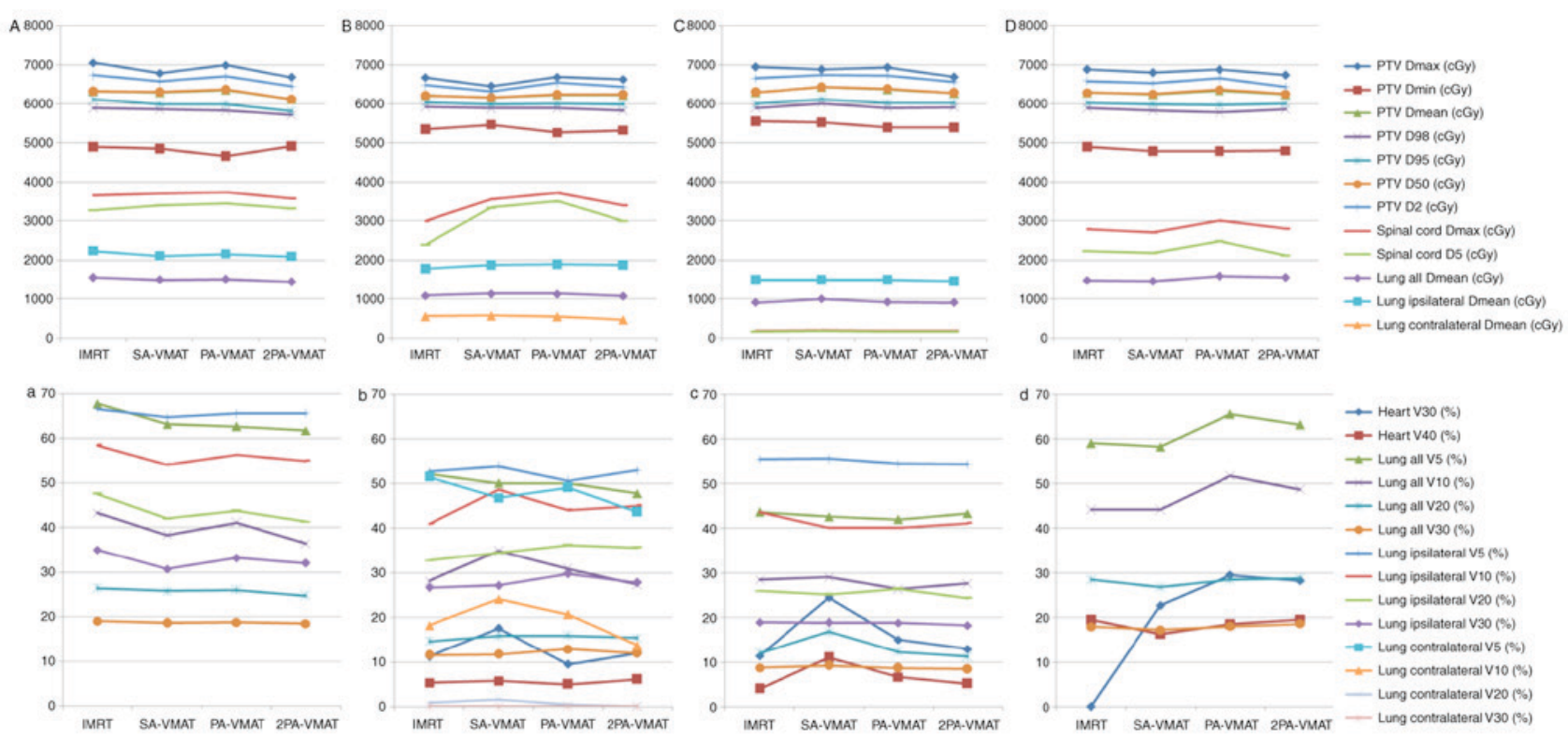

Figure 6. Line charts display the comparison of different dosimetric parameters (indicated by different lines) between VMAT and IMRT plans in 4 patients (indicated by A-a-D-d,) with central lung cancer in which the target volume encompasses the mediastinal lymphatic drainage region. The horizontal axis represents the four different plans. The vertical axis represents dose size. In each case, the capital letters show the unit for dose size in cGy, and the small letters show the unit for dose size in \%. VMAT, volumetric modulated arc therapy; IMRT, intensity-modulated radiation therapy; cGy, centigray; PA, partial arc; SA, single arc; 2PA, double partial arc.

2PA-VMAT plans. This is because the targets were small and far away from normal organs, and radiotherapy plans and dose requirement were easily achieved, resulting in similar $\mathrm{CI}$ and $\mathrm{HI}$ for each plan. Because spinal cords and hearts were far from targets, they received less radiation. V5 (\%) of total lungs in SA-VMAT plans were higher compared with PA-VMAT plans, because the radiation regions in SA-VMAT plans were larger compared with PA-VMAT plans. V5 of total lungs and contralateral lungs in the IMRT plan were higher compared with SA-, PA- and 2PA-VMAT plans. V5 of ipsilateral lungs in IMRT plans were lower compared with SA-, PA- and 2PA-VMAT plans. This was because tumors in most cases located in the lower lobes, so the radiation fields were relatively concentrated and focused to the contralateral lung, which led to higher V5 of contralateral lung and then led to higher V5 of total lungs in IMRT plans. V20 and V30 of total lungs in SA-, PA- and 2PA-VMAT plans were higher compared with IMRT plans. This was because tumors in most cases located near the chest wall, so VMAT plans required a larger range of radiation angles, which resulted in more dispersed dose distribution of 20 and $30 \mathrm{~Gy}$.

In the case of PTV not encompassing the mediastinum in central lung cancer, SA-VMAT plans exhibited better CI and HI than IMRT, PA- and 2PA-VMAT plans. Because the target volumes were relatively large and the target located in the center, the incidence angles of IMRT, PA- and 2PA-VMAT plans were smaller compared with SA-VMAT. However, V30 and V40 of heart in SA-VMAT plans were higher compared with IMRT, PA- and 2PA-VMAT plans. Because the incidence angle of SA-VMAT plan was 360 degree and the target located near the heart, it resulted in the heart receiving a higher radiation dose. The spinal cord was far away from the target thus it received less radiation dose. In most cases, V5, V10 and V20 of total lungs in IMRT plans were higher compared with SA-, 
Table III. Dosage comparison between VMAT and IMRT plans in central lung cancer in which the target volume does not encompass the mediastinal lymphatic drainage region.

\begin{tabular}{|c|c|c|c|c|}
\hline $\begin{array}{l}\text { PTV not encompassing the mediastinal lymphatic } \\
\text { drainage region in central lung cancer }\end{array}$ & $\begin{array}{c}\text { IMRT } \\
(\text { mean } \pm \text { SD) }\end{array}$ & $\begin{array}{l}\text { SA-VMAT } \\
(\text { mean } \pm \text { SD) }\end{array}$ & $\begin{array}{l}\text { PA-VMAT } \\
(\text { mean } \pm \text { SD })\end{array}$ & $\begin{array}{l}\text { 2PA-VMAT } \\
(\text { mean } \pm \mathrm{SD})\end{array}$ \\
\hline PTV Dmax & $6,620.50 \pm 6.55$ & $6,932.80 \pm 6.67$ & $7,015.60 \pm 7.05$ & $6,594.00 \pm 6.56$ \\
\hline PTV Dmin & $5,192.20 \pm 5.55$ & $5,012.90 \pm 5.05$ & $4,897.80 \pm 4.87$ & $5,164.50 \pm 5.11$ \\
\hline PTV D100 (cGy) & $5,192.20 \pm 5.09$ & $5,012.90 \pm 5.00$ & $4,897.80 \pm 4.79$ & $5,164.50 \pm 5.09$ \\
\hline PTV D mean (cGy) & $6,222.90 \pm 6.21$ & $6,506.90 \pm 6.50$ & $6,573.30 \pm 6.53$ & $6,191 \cdot 60 \pm 6.09^{c}$ \\
\hline PTV D98 (cGy) & $5,800.00 \pm 5.79$ & $5,770.00 \pm 5.78$ & $5,700.00 \pm 5.71$ & $5,820.00 \pm 5.88$ \\
\hline PTV D95 (cGy) & $6,000.00 \pm 5.58$ & $6,000.00 \pm 5.59$ & $6,000.00 \pm 5.61$ & $6,000.00 \pm 5.63$ \\
\hline PTV D50 (cGy) & $6,232.00 \pm 6.33$ & $6,232.00 \pm 6.35$ & $6,647.00 \pm 6.65$ & $6,203.00 \pm 6.22$ \\
\hline PTV D2 (cGy) & $6,459.00 \pm 6.49$ & $6,788.00 \pm 6.95$ & $6,901.00 \pm 6.92$ & $6,397.00 \pm 6.41$ \\
\hline Heart V30 $(\%)$ & $14.85 \pm 0.02$ & $16.00 \pm 0.02$ & $25.01 \pm 0.02^{\mathrm{b}}$ & $11.63 \pm 0.02^{\mathrm{c}}$ \\
\hline Heart V40 $(\%)$ & $5.70 \pm 0.02$ & $6.75 \pm 0.02$ & $6.58 \pm 0.02$ & $5.20 \pm 0.02$ \\
\hline Spinalcord D max (cGy) & $4,009.60 \pm 4.02^{\mathrm{a}}$ & $4,389.30 \pm 4.02$ & $4,297.70 \pm 4.02$ & $3,796.00 \pm 3.72^{\mathrm{c}}$ \\
\hline Spinalcord $\mathrm{D}_{5}(\mathrm{cGy})$ & $2,997.00 \pm 2.82$ & $3,300.00 \pm 3.12$ & $3,285.00 \pm 3.13$ & $3,083.00 \pm 3.07$ \\
\hline Lung all V5 $(\%)$ & $49.59 \pm 0.04$ & $50.14 \pm 0.05$ & $49.82 \pm 0.05$ & $50.03 \pm 0.05$ \\
\hline Lung all V10 (\%) & $27.66 \pm 0.02$ & $33.3 \pm 0.03$ & $31.89 \pm 0.03$ & $28.23 \pm 0.02$ \\
\hline Lung all V20 $(\%)$ & $14.83 \pm 0.01$ & $15.25 \pm 0.01$ & $15.28 \pm 0.02$ & $15.01 \pm 0.02$ \\
\hline Lung all V30 (\%) & $11.90 \pm 0.01$ & $11.70 \pm 0.01$ & $11.99 \pm 0.01$ & $12.17 \pm 0.01$ \\
\hline Lung all D mean (cGy) & $1,071.10 \pm 1.02$ & $1,122.40 \pm 1.01$ & $1,136.00 \pm 1.01$ & $1,083.70 \pm 1.02$ \\
\hline Lung-ipsilateral D mean (cGy) & $1,843.80 \pm 1.71$ & $1,892.50 \pm 1.79$ & $1,918.20 \pm 1.91$ & $1,873.50 \pm 1.81$ \\
\hline Lung-ipsilateral V5 (\%) & $53.72 \pm 0.05$ & $53.68 \pm 0.04$ & $52.36 \pm 0.04$ & $52.76 \pm 0.04$ \\
\hline Lung-ipsilateral V10 (\%) & $47.45 \pm 0.04$ & $48.38 \pm 0.04$ & $47.69 \pm 0.04$ & $47.51 \pm 0.04$ \\
\hline Lung-ipsilateral V20 (\%) & $33.38 \pm 0.03$ & $34.74 \pm 0.03$ & $34.59 \pm 0.03$ & $34.73 \pm 0.03$ \\
\hline Lung-ipsilateral V30 (\%) & $27.54 \pm 0.02$ & $27.08 \pm 0.02$ & $27.76 \pm 0.02$ & $28.17 \pm 0.02$ \\
\hline Lung-contralateral D mean (cGy) & $479.90 \pm 0.46$ & $531.20 \pm 0.52$ & $537.00 \pm 0.53$ & $481.20 \pm 0.47^{\mathrm{c}}$ \\
\hline Lung-contralateral V5 (\%) & $46.05 \pm 0.04$ & $46.97 \pm 0.04$ & $47.62 \pm 0.04$ & $47.72 \pm 0.04$ \\
\hline Lung-contralateral V10 & $12.43 \pm 0.01$ & $21.42 \pm 0.02$ & $19.77 \pm 0.01$ & $13.61 \pm 0.01^{\mathrm{c}}$ \\
\hline Lung-contralateral V20 & $0.72 \pm 0.00$ & $0.43 \pm 0.00$ & $0.60 \pm 0.00$ & $0.00 \pm 0.00$ \\
\hline Lung-contralateral V30 & $0.00 \pm 0.00$ & $0.00 \pm 0.00$ & $0.00 \pm 0.00$ & $0.00 \pm 0.00$ \\
\hline $\mathrm{HI}$ & $0.11 \pm 0.00$ & $0.16 \pm 0.00$ & $0.18 \pm 0.00$ & $0.09 \pm 0.00$ \\
\hline $\mathrm{CI}$ & $0.66 \pm 0.00$ & $0.51 \pm 0.00$ & $0.49 \pm 0.00$ & $0.70 \pm 0.00$ \\
\hline
\end{tabular}

${ }^{\mathrm{a}} \mathrm{P}<0.05$ compared with 2PA-VMAT plan; ${ }^{\mathrm{b}} \mathrm{P}<0.05$ compared with $\mathrm{SA}-\mathrm{VMAT}$ plan; ${ }^{\mathrm{c}} \mathrm{P}<0.05$ compared with $\mathrm{SA}-\mathrm{VMAT}$ plan. IMRT, intensity-modulated radiation therapy; VMAT, volumetric modulated arc therapy; SA, single arc; PA, partial arc; 2PA, double partial arc; SD, standard deviation; PTV, planning target volume; cGy, centigray; HI, homogeneity index; CI, conformity index.

PA- and 2PA-VMAT plans, because the target was in the center and the radiation fields of IMRT plans were relatively concentrated. The concentrated radiation fields focused to the ipsilateral lung and resulted in a higher dose. V10 and V20 of total lungs in 2PA-VMAT plans were decreased compared with IMRT, SA- and PA-VMAT plans. The differences of radiation dose of ipsilateral lungs and contralateral lungs were slight.

In the case of PTV encompassing the mediastinum in central lung cancer, the 2PA-VMAT plan in most cases exhibited improved CI and HI compared with IMRT, SA- and PA-VMAT plans. Because the target volume in this case was the largest and the targets were located in the center or near the center, this increased the difficulty of planning. The 2PA-VMAT plan possesses the most optimized incidence angles; therefore it achieved the best CI and HI. Because the optimized incidence angles in 2PA-VMAT plans induced the largest radiation area of heart, V40 of heart in 2PA-VMAT plans were higher compared with IMRT, SA- and 2PA-VMAT plans. Since the spinal cord was near the target, planning optimization for 2PA-VMAT yielded the radiation dose of spinal cord within the tolerance range. V5, V30 of total lungs in 2PA-VMAT plans were higher compared with the IMRT plan, whereas V20 of total lungs in 2PA-VMAT plans were lower compared with IMRT, SA- and PA-VMAT plans. This is because more incidence angles in the 2PA-VMAT plan resulted in increased areas of low dose in contralateral lung and total lungs. In addition, more optimized incidence angles in the 2PA-VMAT plan resulted in lower V20 of total lungs and similar radiation of ipsilateral lung. V5 and V10 of contralateral lungs in SA-, PA- and 2PA-VMAT plans were higher compared with the IMRT plan, whereas V20 and V30 of contralateral lungs in SA-, PA- and 2PA-VMAT plans were lower compared with the 
Table IV. Dosage comparison between VMAT and IMRT plans in central lung cancer in which the target volume encompassing the mediastinal lymphatic drainage region.

\begin{tabular}{|c|c|c|c|c|}
\hline $\begin{array}{l}\text { PTV encompassing the mediastinal lymphatic } \\
\text { drainage region in central lung cancer }\end{array}$ & $\begin{array}{c}\text { IMRT } \\
(\text { mean } \pm \mathrm{SD})\end{array}$ & $\begin{array}{l}\text { SA-VMAT } \\
(\text { mean } \pm \mathrm{SD})\end{array}$ & $\begin{array}{l}\text { PA-VMAT } \\
(\text { mean } \pm \text { SD) }\end{array}$ & $\begin{array}{l}\text { 2PA-VMAT } \\
(\text { mean } \pm \mathrm{SD})\end{array}$ \\
\hline PTV Dmax & $7,063.70 \pm 7.05^{\mathrm{a}}$ & $7,689.30 \pm 7.65$ & $7,651.10 \pm 7.55$ & $7,644.10 \pm 7.55$ \\
\hline PTV Dmin & $4,576.80 \pm 4.55$ & $4,539.10 \pm 4.53$ & $4,325.10 \pm 4.37$ & $4,368.30 \pm 4.38$ \\
\hline PTV D100 (cGy) & $4,576.80 \pm 4.56$ & $4,539.10 \pm 4.51$ & $4,325.10 \pm 4.31$ & $4,368.30 \pm 4.37$ \\
\hline PTV D mean (cGy) & $6,473.90 \pm 6.50^{\mathrm{a}}$ & $6,875.30 \pm 6.79$ & $6,847.40 \pm 6.83$ & $6,725.90 \pm 6.71$ \\
\hline PTV D98 (cGy) & $5,800.00 \pm 5.79$ & $5,800.00 \pm 5.78$ & $5,720.00 \pm 5.72$ & $5,720.00 \pm 5.72$ \\
\hline PTV D95 (cGy) & $6,000.00 \pm 5.58$ & $6,000.00 \pm 5.69$ & $6,000.00 \pm 5.90$ & $6,000.00 \pm 5.68$ \\
\hline PTV D50 (cGy) & $6,513.00 \pm 6.37^{\mathrm{a}}$ & $6,950.00 \pm 6.68$ & $6,948.00 \pm 6.78$ & $6,792.00 \pm 6.68^{c}$ \\
\hline PTV D2 (cGy) & $6,782.00 \pm 6.34^{\mathrm{a}}$ & $7,501.00 \pm 7.24$ & $7,350.00 \pm 7.15$ & $7,198.00 \pm 7.03^{\mathrm{c}}$ \\
\hline Heart V30 $(\%)$ & $43.07 \pm 0.04$ & $36.11 \pm 0.03$ & $42.25 \pm 0.04$ & $42.66 \pm 0.04^{c}$ \\
\hline Heart V40 $(\%)$ & $31.42 \pm 0.03$ & $22.42 \pm 0.02$ & $28.39 \pm 0.02$ & $34.3 \pm 0.03^{\mathrm{c}}$ \\
\hline Spinalcord D max (cGy) & $4,380.3 \pm 4.37^{\mathrm{a}}$ & $4,947.4 \pm 4.87$ & $5,039.6 \pm 5.37$ & $4,515.5 \pm 4.29^{c}$ \\
\hline Spinalcord $\mathrm{D}_{5}(\mathrm{cGy})$ & $3,763.00 \pm 3.39$ & $4,098.00 \pm 4.01$ & $4,175.00 \pm 4.12$ & $3,976.00 \pm 3.97$ \\
\hline Lung all V5e $(\%)$ & $74.67 \pm 0.07$ & $77.31 \pm 0.07$ & $76.43 \pm 0.07$ & $75.93 \pm 0.07$ \\
\hline Lung all V10 (\%) & $63.72 \pm 0.06$ & $70.70 \pm 0.07$ & $67.66 \pm 0.06^{\mathrm{b}}$ & $64.11 \pm 0.06^{c}$ \\
\hline Lung all V20 (\%) & $35.22 \pm 0.03$ & $44.19 \pm 0.04$ & $38.47 \pm 0.03^{\mathrm{b}}$ & $35.18 \pm 0.03^{c}$ \\
\hline Lung all V30 $(\%)$ & $20.54 \pm 0.02^{\mathrm{a}}$ & $22.06 \pm 0.02$ & $23.02 \pm 0.02$ & $21.51 \pm 0.02$ \\
\hline Lung all D mean (cGy) & $1,869.40 \pm 1.78$ & $2,034.40 \pm 2.02$ & $1,968.50 \pm 1.98$ & $1,913.00 \pm 1.99^{c}$ \\
\hline Lung-ipsilateral D mean (cGy) & $33.77 \pm 0.03^{\mathrm{a}}$ & $37.08 \pm 0.03$ & $41.03 \pm 0.03^{\mathrm{b}}$ & $37.26 \pm 0.03$ \\
\hline Lung-ipsilateral V5 (\%) & $80.64 \pm 0.08$ & $82.49 \pm 0.08$ & $80.57 \pm 0.08$ & $80.69 \pm 0.08$ \\
\hline Lung-ipsilateral V10 (\%) & $75.11 \pm 0.07$ & $75.91 \pm 0.07$ & $73.95 \pm 0.07$ & $75.55 \pm 0.07$ \\
\hline Lung-ipsilateral V20 (\%) & $54.19 \pm 0.05$ & $58.49 \pm 0.05$ & $58.27 \pm 0.05$ & $54.60 \pm 0.05$ \\
\hline Lung-ipsilateral V30 (\%) & $33.77 \pm 0.03^{\mathrm{a}}$ & $37.08 \pm 0.03$ & $41.03 \pm 0.03$ & $37.26 \pm 0.03$ \\
\hline Lung-contralateral D mean (cGy) & $1,483.70 \pm 1.37$ & $1,751.90 \pm 1.67$ & $1,540.60 \pm 1.56$ & $1,446.40 \pm 1.46$ \\
\hline Lung-contralateral V5 (\%) & $80.07 \pm 0.08^{a}$ & $86.34 \pm 0.08$ & $86.47 \pm 0.08$ & $84.96 \pm 0.08$ \\
\hline Lung-contralateral V10 & $62.49 \pm 0.06$ & $78.42 \pm 0.07$ & $73.59 \pm 0.07$ & $63.57 \pm 0.06^{\mathrm{c}}$ \\
\hline Lung-contralateral V20 & $20.67 \pm 0.02$ & $36.62 \pm 0.03$ & $23.61 \pm 0.02$ & $20.11 \pm 0.02$ \\
\hline Lung-contralateral V30 & $9.60 \pm 0.00$ & $9.42 \pm 0.00$ & $7.18 \pm 0.00$ & $7.95 \pm 0.00$ \\
\hline $\mathrm{HI}$ & $0.15 \pm 0.00^{\mathrm{a}}$ & $0.24 \pm 0.00$ & $0.23 \pm 0.00$ & $0.22 \pm 0.00$ \\
\hline $\mathrm{CI}$ & $0.70 \pm 0.00^{\mathrm{a}}$ & $0.70 \pm 0.00$ & $0.61 \pm 0.00$ & $0.58 \pm 0.00^{c}$ \\
\hline
\end{tabular}

${ }^{\mathrm{a}} \mathrm{P}<0.05$ compared with $2 \mathrm{PA}-\mathrm{VMAT}$ plan; ${ }^{\mathrm{b}} \mathrm{P}<0.05$ compared with $\mathrm{SA}-\mathrm{VMAT}$ plan; ${ }^{\mathrm{c}} \mathrm{P}<0.05$ compared with $\mathrm{SA}-\mathrm{VMAT}$ plan. IMRT, intensity-modulated radiation therapy; VMAT, volumetric modulated arc therapy; SA, single arc; PA, partial arc; 2PA, double partial arc; $\mathrm{SD}$, standard deviation; PTV, planning target volume; cGy, centigray; HI, homogeneity index; CI, conformity index.

IMRT plan. The reason was the same as the dose comparison of total lungs.

Is the present data demonstrate that it may be necessary to classify the radiotherapy plans of lung cancer into three categories, including peripheral lung cancer, PTV not encompassing mediastinum of central lung cancer, and PTV encompassing mediastinum of central lung cancer. Each of IMRT, SA-VMAT, PA-VMAT, and 2PA-VMAT plan has its individual advantages and therefore it is important to employ different planning techniques regarding to different situations. In peripheral lung cancer, V5 $(\%)$ of the lung in PA-VMAT was low. V20 (\%) and V30 (\%) of the lung in IMRT was low. Different techniques may be used according to different requirements of lung dose. In the case of PTV not encompassing the mediastinum in central lung cancer, the HI and CI of SA-VMAT was the best, but with a relatively high heart dose. The received dose of lung was the highest in the IMRT plan. V10 (\%) of the lung in 2PA-VMAT was the lowest. If the pulmonary function is poor, it may be better to employ 2PA-VMAT instead of IMRT. In case of PTV encompassing mediastinum in central lung cancer, if the PTV is big, it is hard to decide on a good radiotherapy plan. The $\mathrm{HI}$ and $\mathrm{CI}$ of 2PA-VMAT were good but with high heart dose. V5 (\%) and V30 (\%) of the lung in 2PA-VMAT were high, whereas V20 (\%) of the lung was low. Therefore, it may crucial to employ different planning techniques considering different OAR requirements.

Radiation-induced pneumonitis (RIP) is one of the most common and serious complications following radiation of thoracic malignancies, which produces a considerable effect on patient morbidity, even leading to death. The incidence of RIP is closely correlated with the irradiation dose that the 


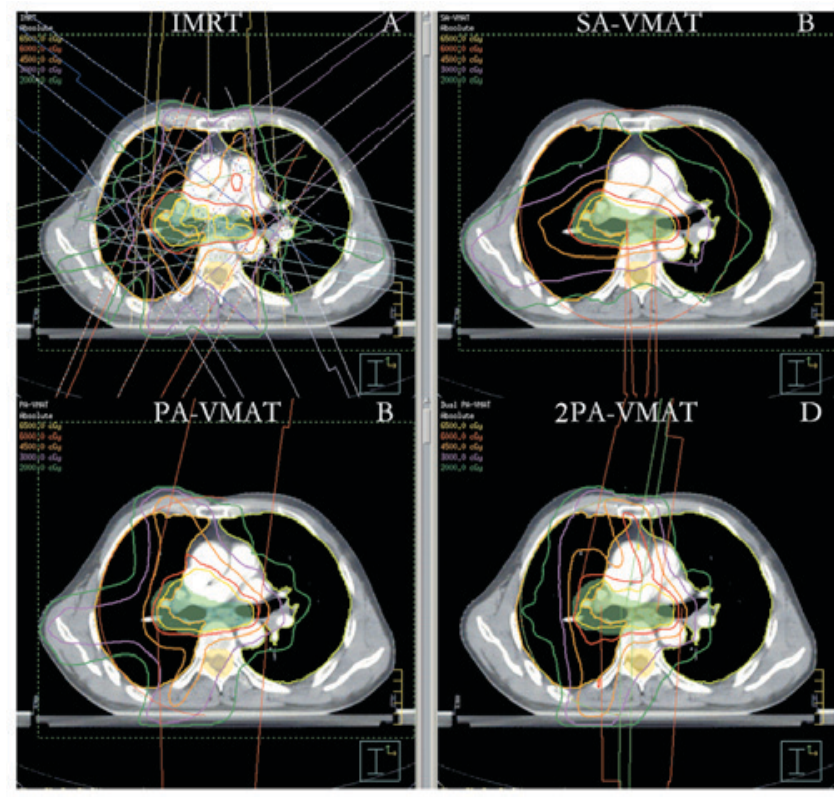

Figure 7. Isodose curves of 2PA-VMAT and IMRT plans in central lung cancer in which the target volume encompasses the mediastinal lymphatic drainage region. (A) Isodose curves of IMRT plan. (B) Isodose curves of SA-VMAT plan. (C) Isodose curves of PA-VMAT plan. (D) Isodose curves of 2PA-VMAT plan. In central lung cancer, when PTV encompasses the mediastinum, 2PA-VMAT exhibited improved PTV coverage compared with IMRT. IMRT exhibited better sparing of spinal cord, lung-all, lung-ipsilateral, and lung-contralateral compared with 2PA-VMAT. In addition, IMRT exhibited better dose conformity and homogeneity. 2PA, double partial arc; VMAT, volumetric modulated arc therapy; IMRT, intensity-modulated radiation therapy; PA, partial arc; SA, single arc; PTV, planning target volume.

lungs received (28). Studies have suggested that the dosimetric parameters of the lung DVH, such as mean lung dose, V20 and V30 of lung, are direct factors which can affect RIP incidence (34-37).

According to Schallenkamp et al (38), a study of a 92-patient cohort, V10/13/15 was also significantly correlated to RIP. Wang et al (39) demonstrated that V5 of both lung lobes was the only parameter predicting the incidence of RIP ( $\geq$ grade 2 ) in NSCLC patients. The present results suggested that VMAT plans obviously delivered low irradiation dose to a larger volume of lung than IMRT, so the VMAT technique may increase RIP incidence more than IMRT in peripheral lung cancer and PTV not encompassing mediastinum of central lung cancer.

Multiple studies have confirmed that VMAT has a significantly shorter delivery time than IMRT (7). The present study also demonstrated that VMAT had a significant advantage of delivery efficiency and treatment time. Improved treatment efficiency of VMAT yielded less scatter dose from reducing MU, which may reduce secondary malignancies. Less treatment time also enhances patient comfort and satisfaction and decreases the intra-fraction variation.

With respect to the present study, no one planning technique was demonstrated to be superior in all aspects. IMRT and 2PA-VMAT plans achieved superior conformal plans in these three kinds of situations compared with the other plans. As for OARs sparing, the four planning techniques could achieve superior sparing of different organs. SA/PA/2PA-VMAT plan were more effective at dose delivery than other plans.
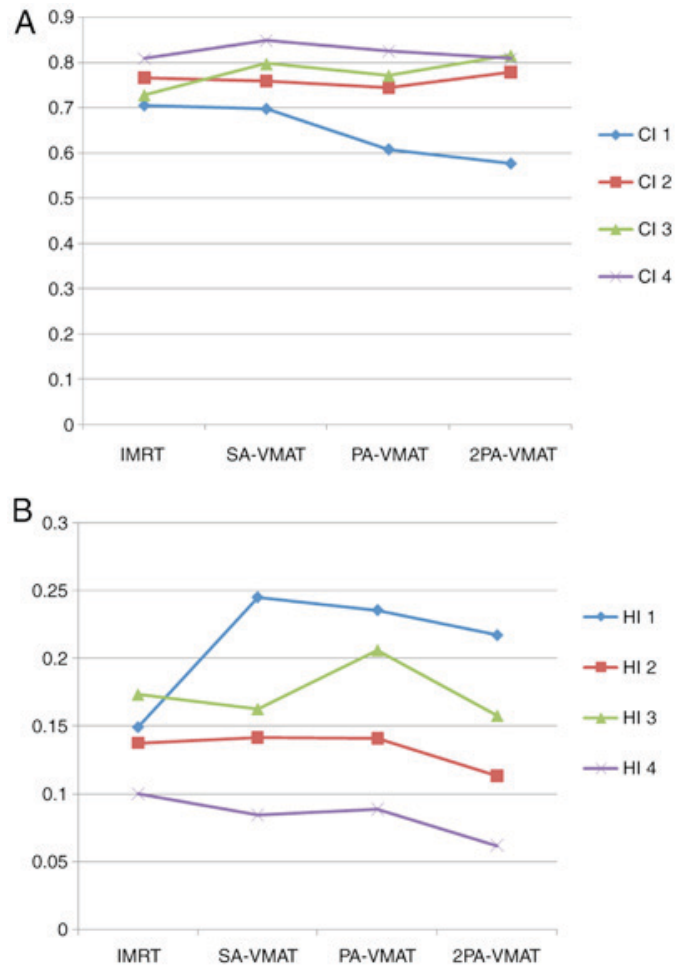

Figure 8. Line charts display the comparison of (A) CI and (B) HI between VMAT and IMRT plans in 4 patients (indicated by different lines) with central lung cancer in which the target volume does not encompass the mediastinal lymphatic drainage region. The horizontal axis represents the four different plans. The vertical axis represents values of CI and HI. CI, conformity index; HI, homogeneity index; VMAT, volumetric modulated arc therapy; IMRT, intensity-modulated radiation therapy; PA, partial arc; SA, single arc; 2PA, double partial arc.

Several studies have suggested that the VMAT plan is better than the IMRT plan in lung cancer. Jiang et al (28) reported that the VMAT plan gets superior PTV coverage than IMRT plans for locally advanced lung cancer. Jiang et al (28) demonstrated that V20, V30 and MLD of the total and contra-lateral lungs in VMAT plans were significantly lower compared with IMRT plans. Other studies have demonstrated that VMAT plans achieved the most objectives on target volumes and OARs for stage III NSCLC $(27,29)$. There may be two reasons which lead to the different results. One reason may be related to different situations of target volume. For example, in the present study, different results were obtained between the case of PTV encompassing mediastinal lymphatic drainage region and the case of PTV not encompassing the mediastinal lymphatic drainage region in central lung cancer. The other reason may be related to different planning system, different accelerator and the effort on planning. The plan quality depends heavily on the amount of effort spent on the planning and planner's experience $(25,40)$. One major challenge of VMAT plans is that VMAT requires a much longer time to optimize compared with IMRT. The long optimization time may introduce more variations in plan quality due to limits on planners' time and effort $(9,11,14)$. Therefore, it is more difficult to assure high-quality of treatment plans for VMAT than for IMRT.

Concrete analysis should be performed according to concrete circumstance of each patient to make the best plan. 

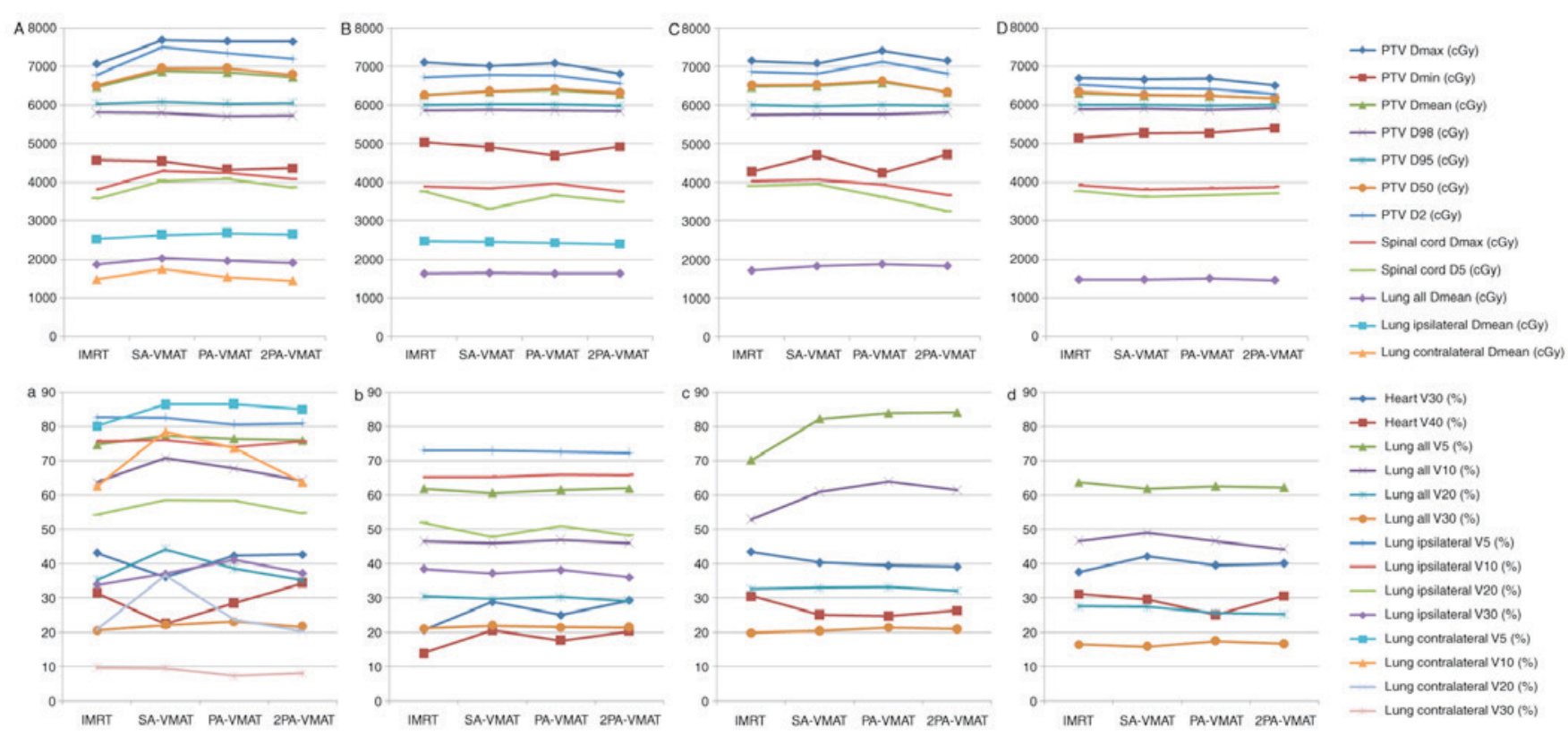

Figure 9. Line charts display the comparison of different dosimetric parameters (indicated by different lines) between VMAT and IMRT plans in 4 patients (indicated by Aa-Dd) with central lung cancer in which the target volume does not encompass the mediastinal lymphatic drainage region. The horizontal axis represents the four different plans. The vertical axis represents dose size. In each case, the capital letters show the unit for dose size in cGy, and the small letters show the unit for dose size in \%. VMAT, volumetric modulated arc therapy; IMRT, intensity-modulated radiation therapy; cGy, centigray; PA, partial arc; SA, single arc; 2PA, double partial arc.

The suitability of the planning technique could vary depending on the staging, tumor size, location of tumor, OARs and the dose-tolerance criteria. However, the data from the present study has limitations, because the small numbers of patients enrolled renders the results less reliable. In addition, for IMRT, alternate planning techniques using more beams could have bene attempted; however, incorporation of such variation could increase the plan complexity at cost of treatment time. Further clinical investigation is required in order to fully address these concerns.

In conclusion, the present results suggest that it may be necessary to classify the radiotherapy plans of lung cancer into three categories, including peripheral lung cancer, PTV not encompassing mediastinum of central lung cancer, and PTV encompassing mediastinum of central lung cancer. In the present study, the dosimetric differences and treatment efficiency were compared between IMRT and VMAT plans in three types of cases. Each of IMRT, SA-VMAT, PA-VMAT, and 2PA-VMAT plan had individual advantages, and it may be crucial to employ different planning techniques in different situations. Different techniques may be used according to different requirements of lung dose. If the pulmonary function is poor, it may be better to employ 2PA-VMAT instead of IMRT. In case of PTV encompassing mediastinum in central lung cancer, if the PTV is large, it may be hard to decide on a good radiotherapy plan. Additionally, it may be important to employ different planning techniques considering different OAR requirements. The suitability of the planning technique could vary depending on the staging, tumor size, location of tumor, OARs as well as the dose-tolerance criteria. Concrete analysis should be made according to concrete circumstance of each patient to make the best plan for radiotherapy of lung cancer.

\section{Acknowledgements}

This study was supported by the National Natural Science Foundation of China (grant nos. 81772793/H1621, $31201060 / \mathrm{C} 0709,30973175 / \mathrm{H} 1621$ and 81172490/H1621), the Program for New Century Excellent Talents in University (grant no. NCET-12-0440), the Scientific and Technological Research Foundation of Shaanxi Province (grant no. 2012K13-01-06), the Research Foundation of Health Department of Shaan'xi Province (grant no. 2010D41), the Qing Nian Jiao Shi Gen Zong Ji Hua of Xi'an Jiaotong University, and a Clinical Research Award of the First Affiliated Hospital of Xi'an Jiaotong University (grant no. XJTU1AHCR2014-041).

\section{References}

1. Peeters ST, Heemsbergen WD, Van Putten WL, Slot A, Tabak H, Mens JW, Lebesque JV and Koper PC: Acute and late complications after radiotherapy for prostate cancer: Results of a multicenter randomized trial comparing 68 to 78 Gy. Int J Radiat Oncol 61: 1019-1034, 2005.

2. Pollack A, Zagars GK, Smith LG, Lee JJ, von Eschenbach AC, Antolak JA, Starkschall G and Rosen I: Preliminary results of a randomized radiotherapy dose-escalation study comparing 70 Gy with 78 Gy for prostate cancer. J Clin Oncol 18: 3904-3911, 2000.

3. Kupelian PA, Ciezki J, Reddy CA, Klein EA and Mahadevan A: Effect of increasing radiation doses on local and distant failures in patients with localized prostate cancer. Int J Radiat Oncol Biol Phys 71: 16-22, 2008.

4. Wolff D, Stieler F, Welzel G, Lorenz F, Abo-Madyan Y, Mai S, Herskind C, Polednik M, Steil V, Wenz F and Lohr F: Volumetric modulated arc therapy (VMAT) vs. serial tomotherapy, step-and-shoot IMRT and 3D-conformal RT for treatment of prostate cancer. Radiother Oncol 93: 226-233, 2009.

5. Brahme A, Roos JE and Lax I: Solution of an integral-equation encountered in rotation therapy. Phys Med Biol 27: 1221-1229, 1982.

6. Bratengeier K: 2-Step IMAT and 2-Step IMRT in three dimensions. Med Phys 32: 3849-3861, 2005. 
7. Otto K: Volumetric modulated arc therapy: IMRT in a single gantry arc. Med Phys 35: 310-317, 2008.

8. Yu CX: Intensity-modulated arc therapy with dynamic multileaf collimation: An alternative to tomotherapy. Phys Med Biol 40: 1435-1449, 1995

9. Palma D, Vollans E, James K, Nakano S, Moiseenko V, Shaffer R, Mckenzie M, Morris J and Otto K: Volumetric modulated arc therapy for delivery of prostate radiotherapy: Comparison with intensity-modulated radiotherapy and three-dimensional conformal radiotherapy. Int J Radiat Oncol Biol Phys 72: 996-1001, 2008.

10. Cozzi L, Dinshaw KA, Shrivastava SK, Mahantshetty U, Engineer R, Deshpande DD, Jamema SV, Vanetti E, Clivio A, Nicolini G and Fogliata A: A treatment planning study comparing volumetric arc modulation with RapidArc and fixed field IMRT for cervix uteri radiotherapy. Radiother Oncol 89: 180-191, 2008.

11. Yoo S, Wu QJ, Lee WR and Yin FF: Radiotherapy treatment plans with RapidArc for prostate cancer involving seminal vesicles and lymph nodes. Int J Radiat Oncol Biol Phys 76: 935-942, 2010.

12. Popescu CC, Olivotto IA, Beckham WA, Ansbacher W, Zavgorodni S, Shaffer R, Wai ES and Otto K: Volumetric modulated arc therapy improves dosimetry and reduces treatment time compared to conventional intensity-modulated radiotherapy for locoregional radiotherapy of left-sided breast cancer and internal mammary nodes. Int J Radiat Oncol Biol Phys 76: 287-295, 2010

13. Clivio A, Fogliata A, Franzetti-Pellanda A, Nicolini G, Vanetti E, Wyttenbach R and Cozzi L: Volumetric-modulated arc radiotherapy for carcinomas of the anal canal: A treatment planning comparison with fixed field IMRT. Radiother Oncol 92: 118-124, 2009.

14. Rao M, Yang WS, Chen F, Sheng K, Ye JS, Mehta V, Shepard D and Cao DL: Comparison of Elekta VMAT with helical tomotherapy and fixed field IMRT: Plan quality, delivery efficiency and accuracy. Med Phys 37: 1350-1359, 2010.

15. Scorsetti M, Bignardi M, Clivio A, Cozzi L, Fogliata A, Lattuada P, Mancosu P, Navarria P, Nicolini G, Urso G, et al: Volumetric modulation arc radiotherapy compared with static gantry intensity-modulated radiotherapy for malignant pleural mesothelioma tumor: A feasibility study. Int J Radiat Oncol Biol Phys 77: 942-949, 2010.

16. Zhang P, Happersett L, Hunt M, Jackson A, Zelefsky M and Mageras G: Volumetric modulated arc therapy: Planning and evaluation for prostate cancer cases. Int J Radiat Oncol Biol Phys 76: 1456-1462, 2010

17. Wu QJ, Yoo S, Kirkpatrick JP, Thongphiew D and Yin FF Volumetric arc intensity-modulated therapy for spine body radiotherapy: Comparison with static intensity-modulated treatment. In J Radiat Oncol Biol Phys 75: 1596-1604, 2009.

18. Zacarias AS, Brown MF and Mills MD: Volumetric modulated arc therapy (VMAT) treatment planning for superficial tumors. Med Dosim 35: 226-229, 2010

19. Verbakel WF, Cuijpers JP, Hoffmans D, Bieker M, Slotman BJ and Senan S: Volumetric intensity-modulated arc therapy vs. conventional IMRT in head-and-neck cancer: A comparative planning and dosimetric study. Int J Radiat Oncol Biol Phys 74: 252-259, 2009.

20. Lagerwaard FJ, Meijer OW, van der Hoorn EA, Verbakel WF, Slotman BJ and Senan S: Volumetric modulated arc radiotherapy for vestibular schwannomas. Int J Radiat Oncol Biol Phys 74: 610-615, 2009

21. Lagerwaard FJ, van der Hoorn EA, Verbakel WF, Haasbeek CJ, Slotman BJ and Senan S: Whole-brain radiotherapy with simultaneous integrated boost to multiple brain metastases using volumetric modulated arc therapy. Int J Radiat Oncol Biol Phys 75: 253-259, 2009

22. Verbakel WF, Senan S, Cuijpers JP, Slotman BJ and Lagerwaard FJ: Rapid delivery of stereotactic radiotherapy for peripheral lung tumors using volumetric intensity-modulated arcs. Radiother Oncol 93: 122-124, 2009.

23. Ong CL, Verbakel WF, Cuijpers JP, Slotman BJ, Lagerwaard FJ and Senan S: Stereotactic radiotherapy for peripheral lung tumors: A comparison of volumetric modulated arc therapy with 3 other delivery techniques. Radiother Oncol 97: 437-442, 2010.

24. Matuszak MM, Yan D, Grills I and Martinez A: Clinical applications of volumetric modulated arc therapy. Int J Radiat Oncol Biol Phys 77: 608-616, 2010.
25. Quan EM, Chang JY, Liao Z, Xia T, Yuan Z, Liu H, Li X, Wages CA, Mohan R and Zhang X: Automated volumetric modulated Arc therapy treatment planning for stage III lung cancer: How does it compare with intensity-modulated radio therapy? Int J Radiat Oncol Biol Phys 84: e69-e76, 2012.

26. van't Riet A, Mak AC, Moerland MA, Elders LH and van der Zee W: A conformation number to quantify the degree of conformality in brachytherapy and external beam irradiation: Application to the prostate. Int J Radiat Oncol Biol Phys 37: 731-736, 1997.

27. Scorsetti M, Navarria P, Mancosu P, Alongi F, Castiglioni S, Cavina R, Cozzi L, Fogliata A, Pentimalli S, Tozzi A and Santoro A: Large volume unresectable locally advanced non-small cell lung cancer: Acute toxicity and initial outcome results with rapid arc. Radiat Oncol 5: 94, 2010.

28. Jiang X, Li T, Liu Y, Zhou L, Xu Y, Zhou X and Gong Y: Planning analysis for locally advanced lung cancer: Dosimetric and efficiency comparisons between intensity-modulated radiotherapy (IMRT), single-arc/partial-arc volumetric modulated arc therapy (SA/PA-VMAT). Radiat Oncol 6: 140, 2011.

29. McGrath SD, Matuszak MM, Yan D, Kestin LL, Martinez AA and Grills IS: Volumetric modulated arc therapy for delivery of hypofractionated stereotactic lung radiotherapy: A dosimetric and treatment efficiency analysis. Radiother Oncol 95: 153-157, 2010.

30. Holt A, van Vliet-Vroegindeweij C, Mans A, Belderbos JS and Damen EM: Volumetric-modulated arc therapy for stereotactic body radiotherapy of lung tumors: A comparison with intensity-modulated radiotherapy techniques. Int J Radiat Oncol Biol Phys 81: 1560-1567, 2011.

31. Bertelsen A, Hansen CR, Johansen J and Brink C: Single Arc volumetric modulated Arc therapy of head and neck cancer. Radiother Oncol 95: 142-148, 2010.

32. Guckenberger M, Richter A, Krieger T, Wilbert J, Baier K and Flentje M: Is a single arc sufficient in volumetric-modulated arc therapy (VMAT) for complex-shaped target volumes? Radiother Oncol 93: 259-265, 2009.

33. Shaffer R, Morris WJ, Moiseenko V, Welsh M, Crumley C, Nakano S, Schmuland M, Pickles T and Otto K: Volumetric modulated Arc therapy and conventional intensity-modulated radiotherapy for simultaneous maximal intraprostatic boost: A planning comparison study. Clin Oncol (R Coll Radiol) 21: 401-407, 2009.

34. Hernando ML, Marks LB, Bentel GC, Zhou SM, Hollis D, Das SK, Fan M, Munley MT, Shafman TD, Anscher MS and Lind PA: Radiation-induced pulmonary toxicity: A dose-volume histogram analysis in 201 patients with lung cancer. Int J Radiat Oncol Biol Phys 51: 650-659, 2001.

35. Claude L, Pérol D, Ginestet C, Falchero L, Arpin D, Vincent M, Martel I, Hominal S, Cordier JF and Carrie C: A prospective study on radiation pneumonitis following conformal radiation therapy in non-small-cell lung cancer: Clinical and dosimetric factors analysis. Radiother Oncol 71: 175-181, 2004.

36. Rancati T, Ceresoli GL, Gagliardi G, Schipani S and Cattaneo GM: Factors predicting radiation pneumonitis in lung cancer patients: A retrospective study. Radiother Oncol 67: 275-283, 2003.

37. Graham MV, Purdy JA, Emami B, Harms W, Bosch W, Lockett MA and Perez CA: Clinical dose-volume histogram analysis for pneumonitis after 3D treatment for non-small cell lung cancer (NSCLC). Int J Radiat Oncol Biol Phys 45: 323-329, 1999.

38. Schallenkamp JM, Miller RC, Brinkmann DH, Foote T and Garces YI: Incidence of radiation pneumonitis after thoracic irradiation: Dose-volume correlates. Int J Radiat Oncol Biol Phys 67: 410-416, 2007.

39. Wang S, Liao Z, Wei X, Liu HH, Tucker SL, Hu CS, Mohan R, Cox JD and Komaki R: Analysis of clinical and dosimetric factors associated with treatment-related pneumonitis (TRP) in patients with non-small-cell lung cancer (NSCLC) treated with concurrent chemotherapy and three-dimensional conformal radiotherapy (3D-CRT). Int J Radiat Oncol Biol Phys 66: 1399-1407, 2006.

40. Chung HT, Lee B, Park E, Lu JJ and Xia P: Can all centers plan intensity-modulated radiotherapy (IMRT) effectively? An external audit of dosimetric comparisons between three-dimensional conformal radiotherapy and IMRT for adjuvant chemoradiation for gastric cancer. Int J Radiat Oncol Biol Phys 71: 1167-1174, 2008.

This work is licensed under a Creative Commons Attribution-NonCommercial-NoDerivatives 4.0 International (CC BY-NC-ND 4.0) License. 